\title{
Cigarette Filter Ventilation and Smoking Protocol Influence Aldehyde Smoke Yields
}

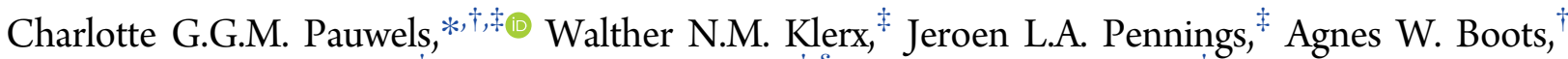 \\ Frederik J. van Schooten, ${ }^{\dagger}$ Antoon Opperhuizen, ${ }^{\dagger, \S}$ and Reinskje Talhout ${ }^{\ddagger}$
}

\begin{abstract}
${ }^{\dagger}$ Department of Pharmacology and Toxicology, NUTRIM School of Nutrition and Translational Research in Metabolism, Maastricht University, P.O. Box 616, 6200 MD Maastricht, The Netherlands

${ }^{\ddagger}$ Centre for Health Protection, National Institute for Public Health and the Environment (RIVM), P.O. Box 1, 3720 BA Bilthoven, The Netherlands

${ }^{\S}$ Office of Risk Assessment and Research, Netherlands Food and Consumer Product Safety Authority (NVWA), P.O. Box 8433, 3503 RK Utrecht, The Netherlands
\end{abstract}

Supporting Information

\begin{abstract}
The WHO study group on tobacco product regulation (TobReg) advised regulating and lowering toxicant levels in cigarette smoke. Aldehydes are one of the chemical classes on the TobReg smoke toxicants priority list. To provide insight in factors determining aldehyde yields, the levels of 12 aldehydes in mainstream cigarette smoke of 11 Dutch brands were quantified. Variations in smoking behavior and cigarette design affecting human exposure to aldehydes were studied by using four different machine testing protocols. Machine smoking was based on the International Standardization Organization (ISO) and Health Canada Intense (HCI) regime, both with and without taping the filter vents. The 11 cigarette brands differed in (i) design and

Acetaldehyde smoke yields of 11 Dutch cigarette brands are significantly higher when machine smoked with the $\mathrm{HCl}$ method, as compared to the ISO method.

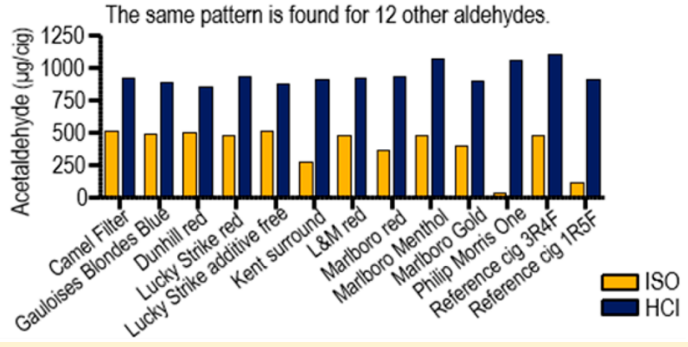
blend characteristics; (ii) tar, nicotine, and carbon monoxide (TNCO) levels; (iii) popularity; and (iv) manufacturer. Cigarette smoke was trapped on a Cambridge filter pad and carboxen cartridge. After being dissolved in methanol/CS $\mathrm{CS}_{2}$ and derivatization with $\mathrm{DNPH}$, the aldehyde yields were determined using HPLC-DAD. Using an intense smoking regime (increased puff volume, shorter puff interval) significantly increased aldehyde yields, following the pattern: ISO < ISO-taped < HCI-untaped < HCI. For all of the regimes, acetaldehyde and acrolein yields were strongly correlated $(r=0.804)$. The difference in TNCO and aldehyde levels between regular and highly ventilated low-TNCO cigarettes (as measured using ISO) diminished when smoking intensely; this effect is stronger when combined with taping filter vents. The highly ventilated low-TNCO brands showed six times more aldehyde production per mg nicotine for the intense smoking regimes. In conclusion, acetaldehyde and acrolein can be used as representatives for the class of volatile aldehydes for the different brands and smoking regimes. The aldehyde-to-nicotine ratio increased when highly ventilated cigarettes were smoked intensely, similar to real smokers. Thus, a smoker of highly ventilated low-TNCO cigarettes has an increased potential for higher aldehyde exposures compared to a smoker of regular cigarettes.
\end{abstract}

\section{INTRODUCTION}

During the Framework Convention on Tobacco Control (FCTC), parties started to develop guidelines for the regulation of contents and emissions of tobacco products in order to reduce tobaccorelated morbidity and mortality. ${ }^{1}$ As part of this, the WHO study group on tobacco product regulation (TobReg) advised regulating and lowering toxicant yields in cigarette smoke. ${ }^{2}$ Due to the complex composition of cigarette smoke and the wide variety of tobacco-related diseases, TobReg proposed beginning regulation with a limited set of high-priority toxic emissions. To this end, priority lists of smoke toxicants were compiled based on toxicity indices in combination with other information on products. This indicates that regulation is feasible, for example, the considerable differences in emission levels between brands under similar smoking conditions. ${ }^{2-6}$ One of the chemical classes identified by TobReg, as well as others, as a priority, due to their impact on human health, is the volatile aldehydes. ${ }^{1,2,4,7,8}$ In order to study their adverse health effects, modeled human exposures to cigarette-derived aldehydes were compared to no observed adverse effect levels (NOAELs) or the lowest observed adverse effect levels (LOAELs) derived from animal experimental data, which was translated to human risks using safety factors. Risk assessors concluded that exposure to individual aldehydes leads to adverse acute and chronic health effects. ${ }^{6,9-11}$ Burns et al. showed that for all brand characteristics and human smoking behaviors, ${ }^{2}$ the chemical class of aldehydes present in cigarette smoke may be represented by only three compounds,

Received: December 22, 2017

Published: May 4, 2018 
that is, acetaldehyde, formaldehyde, and acrolein. These three were selected because of similarities in structures, precursors, or mechanisms of formation. ${ }^{2}$ It is suggested to express the toxicant yields per $\mathrm{mg}$ of nicotine, since smokers titrate the nicotine need.

Since smoking behavior varies between individuals, it is important to study the influence of smoking behavior on individual exposure to cigarette mainstream smoke (MSS) constituents. Machine smoking with standardized settings for puff volume, puff duration, and interpuff intervals can be used to create a range of mimicked smoking intensities that cover exposure for all human smokers. For this purpose, the International Organization for Standardization (ISO) smoking regime (puff volume $35 \mathrm{~mL}$, puff duration $2 \mathrm{~s}$, puff interval $60 \mathrm{~s}$ ) and Health Canada Intense (HCI) (puff volume $55 \mathrm{~mL}$, puff duration $2 \mathrm{~s}$, puff interval $30 \mathrm{~s}$ ) smoking regimes are often applied. In addition to the HCI smoking regime being more intense, another difference with the ISO smoking regime is that the cigarette filters are taped to block filter vents. Industry uses cigarette filter ventilation and cigarette paper porosity as a major design feature, resulting in reduced yields of tar, nicotine, and carbon monoxide $(\mathrm{CO})$ as measured using the ISO regime. ${ }^{12}$ This is explained by the dilution of the emissions produced in the burning tip, with ambient air invading via filter vents before the emissions are collected at the mouth end. The influence of cigarette filter ventilation is determined by combining the smoking regimes and blocking filter vents with tape. ${ }^{13,14}$ It has been shown that machine-smoked cigarettes produce significantly lower yields per puff and per cigarette when ISO regimes are applied rather than HCI conditions. ${ }^{15}$ On the one hand, filter ventilation is responsible for large differences in aldehyde yields under machine smoking conditions, ${ }^{16}$ explained by a changed burning process. $^{17,18}$ On the other hand, due to the increased puff parameters, additional smoke is produced. The combination of filter ventilation and puff parameters is investigated in four smoking conditions.

The industry aims at increasing product elasticity to facilitate the ability of smokers to extract desirable levels of nicotine. ${ }^{19}$ A good example is the low-TNCO cigarette, which smokers unconsciously manipulate in such a way that they extract enough nicotine to satisfy their needs. ${ }^{20-22}$ In addition to smoking behavior and filter ventilation, other brand characteristics also influence smoke constituent yields, such as aldehyde yields. A cigarette brand is unique because of agricultural practices, plant characteristics, tobacco blending, and cigarette design. ${ }^{23}$ The curing process and the type and blend of tobacco, i.e. Virginia/Bright, Oriental, and Burley tobacco, are important contributors to the aldehyde yields in cigarette smoke. ${ }^{3,16}$ Natural sugars in tobacco leaves are thought to be a significant source of aldehydes. ${ }^{11,24}$ In addition, sugar is added by the manufacturer to improve the flavor and taste of the smoke. ${ }^{25-27}$ It has already been shown that lowering sugar content is promising for reducing aldehydes in smoke. ${ }^{11,28}$

The objectives of the present study are:

(1) to quantify aldehyde levels in cigarette MSS for four different smoking regimes and 11 different brands;

(2) to study whether acrolein, acetaldehyde, and formaldehyde levels correlate well with the other aldehyde levels and are thus representative of the class of volatile aldehydes;

(3) to quantify TNCO levels for quality assurance purposes and to be able to estimate nicotine exposure;
(4) to estimate the influence of the smoking regime, especially filter ventilation, and brand characteristics on the aldehyde yields in cigarette smoke;

(5) and to estimate the aldehyde exposure of a smoker for different brands and smoking regimes.

\section{EXPERIMENTAL PROCEDURES}

Eleven cigarette brands, available on the Dutch market in 2015, were selected based on different TNCO levels, popularity, tobacco blend, and cigarette manufacturer (Table 1). According to the website of the tobacco company, the selected cigarettes in the main study typically contain the three main tobacco types in different ratios: Virginia/ Bright, Burley, and Oriental. All of the brands used were bought at a tobacconist and have the same batch code on the package. Cigarettes were conditioned and marked as described in ISO 3402:1999 and ISO $4387 .{ }^{29,30}$ In addition, two reference cigarettes $3 \mathrm{R} 4 \mathrm{~F}$ and $1 \mathrm{R} 5 \mathrm{~F}$, with a high and low TNCO level respectively, were purchased from the College of Agriculture Reference Cigarette Program, University of Kentucky (Lexington, KY 40546, USA).

Study Design. Cigarettes were machine-smoked on a 20-port linear smoking machine (Cerulean SM450, Milton Keynes, U.K.), according to the ISO and HCI smoking regimes as described by ISO 3308:2012 and WHO TobLabNet SOP 01. ${ }^{31,32}$ The ISO smoking regime was based on a $35 \mathrm{~mL}$ puff volume, $2 \mathrm{~s}$ puff duration, $60 \mathrm{~s}$ puff interval, and no vent blocking. The HCI smoking regime was described as $55 \mathrm{~mL}$ puff volume, $2 \mathrm{~s}$ puff duration, and $30 \mathrm{~s}$ puff interval. A $100 \%$ vent blocking was achieved by taping the vents with $19 \mathrm{~mm}$ width Scotch Magic tape (cat no: 810, 3M, USA). Two extra smoking regime variations on the conventional methods were included: the ISO method with filter vent blocking by taping (ISO-taped) and the HCI method without taping the filter vents (HCI-untaped).

Aldehyde Measurements. The $85.2 \mathrm{mmol} / \mathrm{L}$ 2,4-dinitrophenylhydrazine hydrochloride (DNPH) reagent was composed of hydrochloride, phosphoric acid (85\%) (Merck), and acetonitrile (>99\%) (Biosolve). Calibration standards were made for formaldehyde-DNPH (97\%) (Sigma-Aldrich), acetaldehyde-DNPH (Sigma-Aldrich), acrolein-DNPH (Sigma-Aldrich), including 0, 0.1, 0.2, 0.5, 1.0, and $2.0 \mu \mathrm{g} / \mathrm{mL}$, and an aldehyde/ketone-DNPH mix (ERA-020, Sigma-Aldrich), including $0,0.15,0.45,0.90,1.80$, and $3.0 \mu \mathrm{g} / \mathrm{mL}$.

For the aldehyde measurement, cigarette smoke was trapped on a holder containing a Cambridge filter pad (CFP) and a CX 572 20/45 cartridge (cat no: $11072-\mathrm{U}$, Sigma) $300 \mathrm{mg}$ in a $3 \mathrm{~mL}$ SPE tube (cat no: 57241, Sigma). Extraction was done as described in the WHO SOP08. ${ }^{33}$ In short, CFP and carboxen were extracted by $10 \mathrm{~mL}$ of carbon disulfide/methanol (20/80), while being shaken for $10 \mathrm{~min}$ at $120 \mathrm{rpm}$. A half of a milliliter was taken to derivate with $0.2 \mathrm{~mL}$ of DNPH solution for $10 \mathrm{~min}$, followed by a dilution with $4.3 \mathrm{~mL}$ of ethanol. The aldehydes were quantified by HPLC LC-10Ai (Shimadzu), including a SIL-20AC autosampler and a SPD-M20A photo diode array detector, quantified at wavelength $360 \mathrm{~nm}$, and verification of identity wavelength scan was done between 250 and $450 \mathrm{~nm}$. As a mobile phase, a gradient of water (A) and acetonitrile (B) was used, starting with A 55\% and B 45\%, after 25 min it changed to B $100 \%$, with a change back to A $55 \%$ and B $45 \%$ after $31 \mathrm{~min}$, and ending the run at $45 \mathrm{~min}$. The individual aldehydes were measured in five replicates per cigarette brand, using four smoking conditions. Aldehydes of interest were acetaldehyde, acrolein, formaldehyde, acetone, propionaldehyde, crotonaldehyde, butyraldehyde, benzaldehyde, isovaleraldehyde, hexaldehyde, and 2,5-dimethylbenzaldehyde, with $o-, m$-, and $p$-tolualdehyde combined. The latter compound was determined as a single compound since the chromatographic method did not separate the three isomers.

TNCO Measurements. TNCO in cigarette smoke was measured under the same conditions as the aldehyde measurements. A $44 \mathrm{~mm}$ diameter CFP (product no: 9703-9654, Whatman, GE Healthcare U.K. Limited) was used to trap the mainstream cigarette smoke constituents and was immediately extracted with isopropanol (>99.8\%) (CAS 67-63-0, Merck), containing heptadecane (99.6\%) (CAS 629-78-7, Sigma-Aldrich) as the internal standard for nicotine 




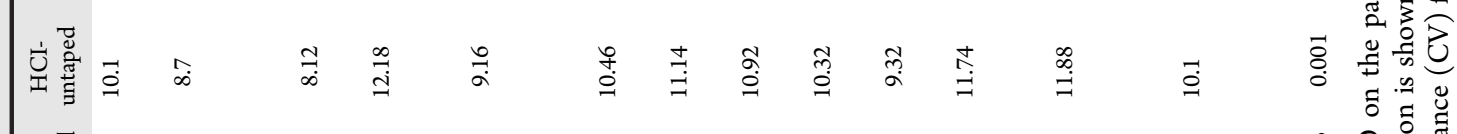



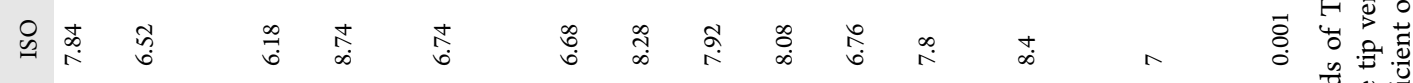

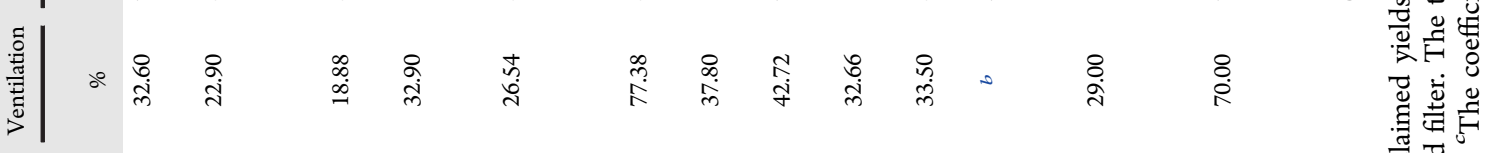

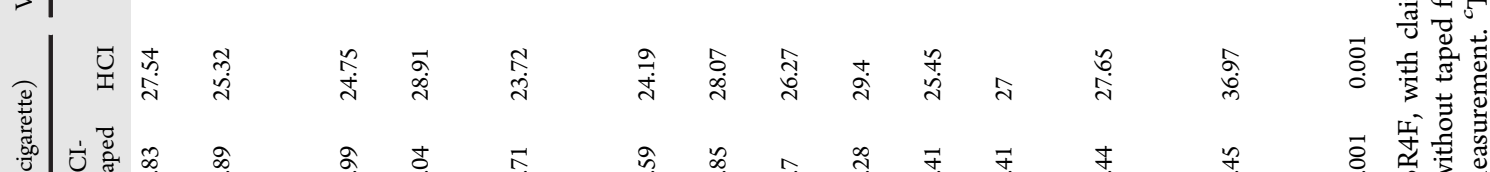

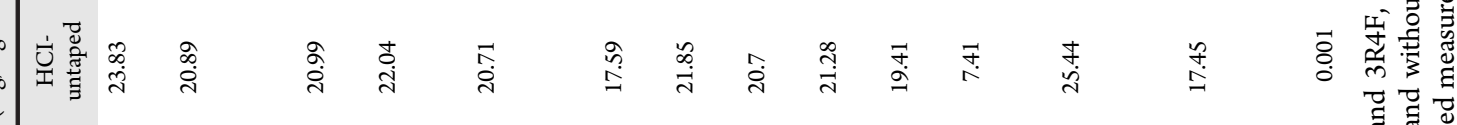

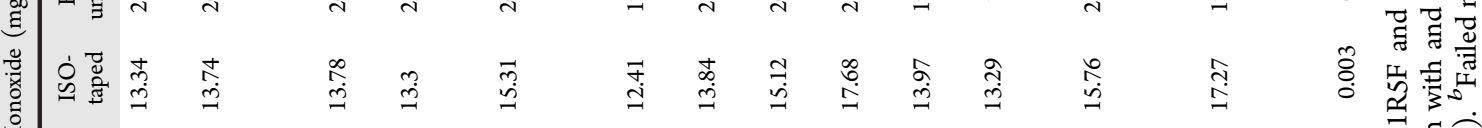

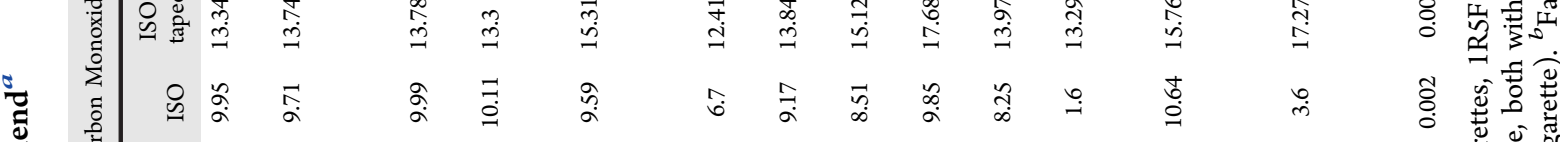
衰 $\varrho \varrho \varrho$

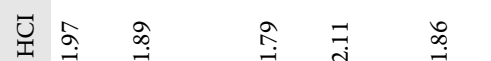

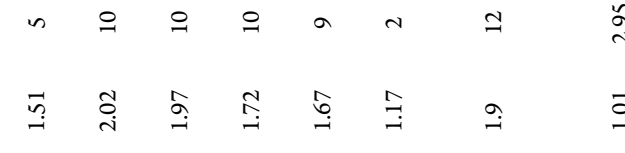

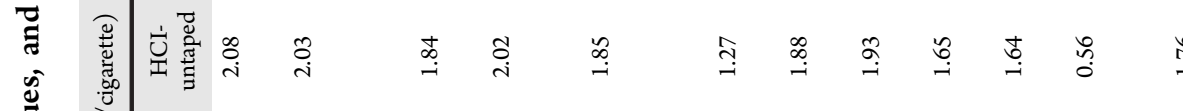



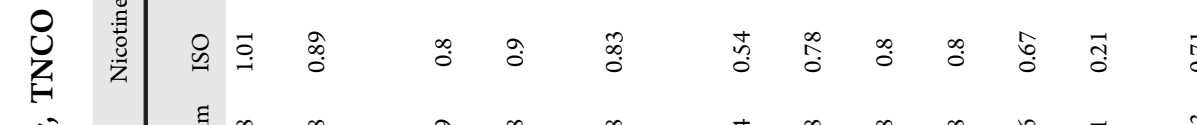

\section{然}

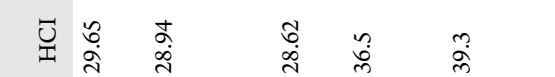

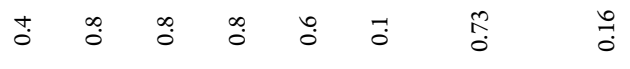

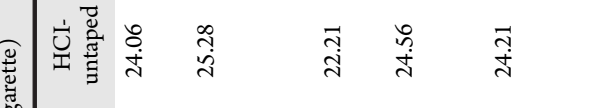

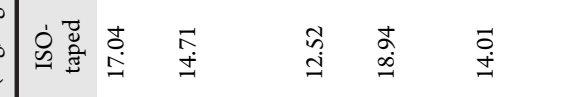

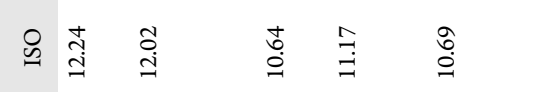

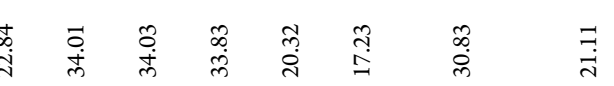

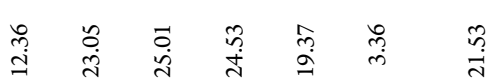

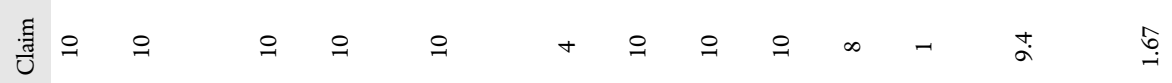

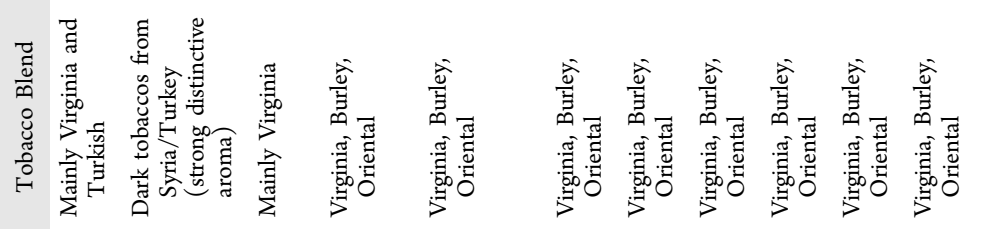

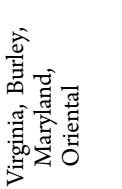

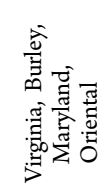

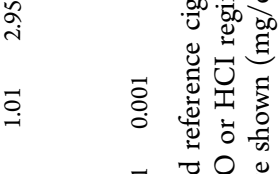

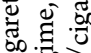

(大)

?ํ.

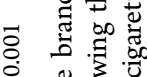

害 응

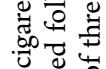

部

घ

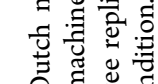

을

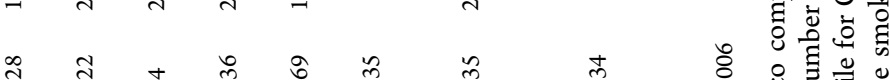

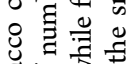

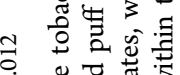

菏.

范 0

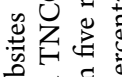

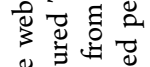

政

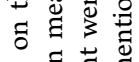

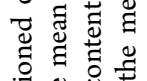

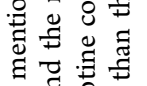



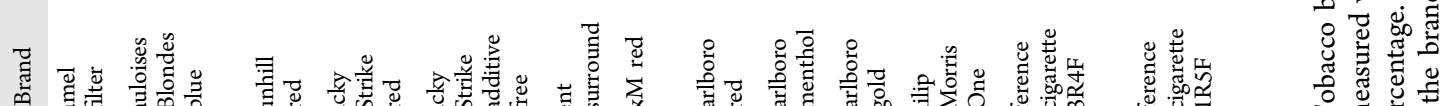



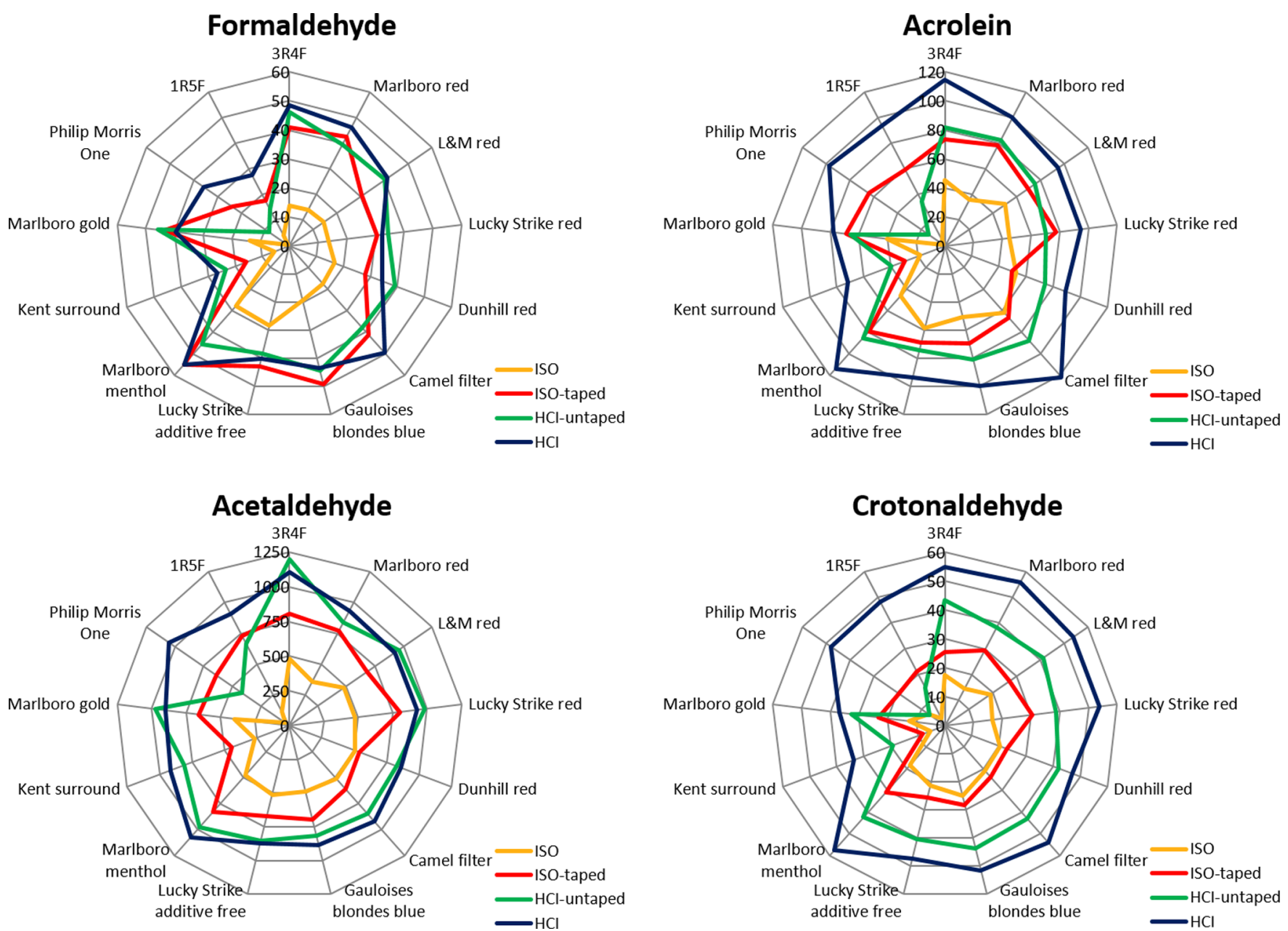

Figure 1. Radar chart shows cigarette smoke emissions of formaldehyde, acrolein, acetaldehyde, and crotonaldehyde (mean $\mu \mathrm{g} / \mathrm{cigarette})$ of 13 different cigarette brands when machine-smoked according to the four different smoking conditions $(n=5)$.

(Art18142, Across). Ethanol (Merck) was used as an internal standard for water. Smoke extracts were analyzed by GC flame ionization and thermal conductivity detectors, with the use of WCOT fused silica coated with CP-WAX 51 for amines $25 \mathrm{~m} \times 0.25 \mathrm{~mm}$ ID (part CP7405, Agilent Technologies) and PoraBond Q $25 \mathrm{~m} \times 0.32 \mathrm{~m} \mathrm{ID}$, $5 \mu \mathrm{m}$ (part CP7351I5, Agilent Technologies). Tar, nicotine, and CO concentrations were determined as described in ISO 4387:2000, ISO 10315:2014, and ISO 8454:2007, with a modification for the tar and nicotine measurements of one cigarette per CFP and for the CO measurements of three cigarettes per smoking session and five and three replicates for four smoking conditions, respectively. ${ }^{30,34,35}$

Filter Ventilation. Filter tip ventilation is the percentage of smoke that is diluted by air when a smoker takes a puff. The KC-3 apparatus (Borgwaldt-KC, Richmond, Virginia, USA) was used to measure the filter tip ventilation at a flow rate of $17.5 \mathrm{~mL} / \mathrm{s}$ for five cigarettes per brand. ${ }^{36}$

Statistical Analysis. Aldehyde yields in cigarette smoke were calculated as $\mu \mathrm{g}$ per cigarette and TNCO as mg per cigarette. Mean yields and standard deviations were given for all components per cigarette brand and smoking condition. To compare the degree of variation between samples, the coefficient of variance was calculated for TNCO. Values for smoke components were compared using ANOVA statistics. For comparison between brands within each single smoking condition, one-way ANOVA was used. For comparison between brands and smoking conditions, two-way ANOVA was used, with brands and smoking condition as factors. Differences were considered significantly different when $p<0.05$. Correlations were determined between the individual aldehydes as the Pearson correlation coefficient.

\section{RESULTS}

Aldehyde Levels in Cigarette Mainstream Smoke. The smoke emissions of several aldehydes from different cigarette brands were measured using the machine smoking regimes ISO and HCI, both with and without the ventilation holes taped (Figure 1, Table S2). The different brands, the manufacturer, the claimed and measured TNCO values, and the tobacco blend are listed in Table 1. For both regimes, fewer puffs were taken per cigarette when the ventilation holes were taped, but the number of puffs per cigarette increased when ISO was compared to HCI.

Significantly different yields of individual aldehyde emissions were produced for both different cigarette brand characteristics and different smoking conditions $\left(p<1 \times 10^{-9}\right.$ in all cases) (Table S3). Within one of the four smoking conditions, taking the cigarette brands as the only variable into account, the individual aldehydes show significant differences in yield when compared to each other $(p<0.02)$.

Aldehyde yields significantly increased when the smoking regime was intensified (increasing puff volume and puff interval) (Table S3). Nearly all cigarette smoke emissions of aldehydes showed the same pattern, as illustrated in Figure 1, ISO $<$ ISOtaped $<$ HCI-untaped $<$ HCI. Apart from the highly ventilated cigarettes Philip Morris One and 1R5F, this pattern is not observed for formaldehyde. While differences between ISO, on the one hand, and ISO-taped, HCI-untaped, and HCI, on the other, are clearly noticeable, differences between ISO-taped, 
Table 2. Correlation Coefficients of Individual Aldehyde Emissions Produced by 13 Cigarette Brands (Five Replicates) across the Combined Smoking Conditions

\begin{tabular}{|c|c|c|c|c|c|c|c|c|c|c|c|c|c|}
\hline son correlation & 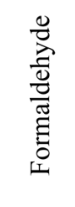 & $\begin{array}{l}\frac{0}{0} \\
\frac{d}{0} \\
\frac{0}{0} \\
\frac{\pi}{0} \\
\frac{0}{4}\end{array}$ & $\frac{\frac{\Xi}{0}}{\frac{0}{0}}$ & $\begin{array}{l}\stackrel{0}{0} \\
\stackrel{0}{0} \\
\stackrel{0}{U}\end{array}$ &  & 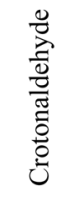 & 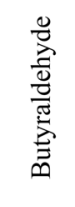 & 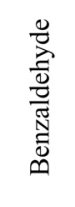 & $\begin{array}{l}\frac{\theta}{2} \\
\frac{\pi}{0} \\
\frac{0}{\pi} \\
\frac{\pi}{\pi} \\
\frac{\pi}{0} \\
0\end{array}$ & 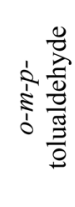 & 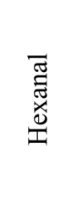 & 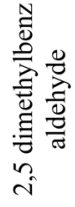 & $\begin{array}{l}\frac{0}{5} \\
\frac{5}{5} \\
\frac{0}{0} \\
0\end{array}$ \\
\hline Formaldehyde & - & 614 & 0.576 & 0.438 & 530 & 0.553 & 566 & 0.506 & 0.507 & 0.594 & 0.579 & 0.591 & \\
\hline Acetaldehyde & 0.614 & - & 0.804 & 0.775 & 0.868 & 0.831 & 0.909 & 0.818 & 0.874 & 0.786 & 0.698 & 0.849 & 0.0 \\
\hline Acrolein & 0.576 & 0.804 & - & 0.807 & 0.921 & 0.896 & 0.886 & 0.734 & 0.850 & 0.744 & 0.564 & 0.768 & 0.1 \\
\hline Acetone & 0.438 & 0.775 & 0.807 & - & 0.863 & 0.832 & 0.851 & 0.740 & 0.847 & 0.669 & 0.500 & 0.780 & 0.2 \\
\hline Propanal & 0.530 & 0.868 & 0.921 & 0.863 & - & 0.965 & 0.977 & 0.902 & 0.968 & 0.840 & 0.678 & 0.880 & 0.3 \\
\hline Crotonaldehyde & 0.553 & 0.831 & 0.896 & 0.832 & 0.965 & - & 0.958 & 0.903 & 0.964 & 0.803 & 0.666 & 0.908 & 0.4 \\
\hline Butyraldehyde & 0.566 & 0.909 & 0.886 & 0.851 & 0.977 & 0.958 & - & 0.927 & 0.977 & 0.857 & 0.751 & 0.923 & 0.5 \\
\hline Benzaldehyde & 0.506 & 0.818 & 0.734 & 0.740 & 0.902 & 0.903 & 0.927 & - & 0.935 & 0.816 & 0.770 & 0.911 & 0.6 \\
\hline Isovaleraldehyde & 0.507 & 0.874 & 0.850 & 0.847 & 0.968 & 0.964 & 0.977 & 0.935 & - & 0.842 & 0.704 & 0.923 & 0.7 \\
\hline$o-m-p$-tolualdehyde & 0.594 & 0.786 & 0.744 & 0.669 & 0.840 & 0.803 & 0.857 & 0.816 & 0.842 & - & 0.875 & 0.798 & 0.8 \\
\hline Hexanal & 0.579 & 0.698 & 0.564 & 0.500 & 0.678 & 0.666 & 0.751 & 0.770 & 0.704 & 0.875 & - & 0.741 & 0.9 \\
\hline $\begin{array}{r}\text { 2,5 dimethylbenz } \\
\text { aldehyde }\end{array}$ & 0.591 & 0.849 & 0.768 & 0.780 & 0.880 & 0.908 & 0.923 & 0.911 & 0.923 & 0.798 & 0.741 & - & 1.0 \\
\hline
\end{tabular}

HCI-untaped, and HCI are not so clear. To a lesser extent, this is also the case for acetaldehyde, where differences between HCI-untaped and HCI are absent for most brands.

The other individual aldehydes show comparable increases in yield due to intensifying the smoking regime or taping the filter vents (Table S2). There were exceptions for acetone when using ISO-taped and for hexaldehyde and $o$-, $m$-, and $p$-tolualdehyde when using HCI.

In summary, the individual aldehyde yields increase as a result of the smoking regime and the filter vents being taped.

Correlation between Aldehyde Levels. Additionally, the relation between the different aldehydes that are present in the cigarette smoke was investigated (Table 2, Figure 2). It was found that the different aldehydes produced in different cigarette brands correlate well within a particular smoking regime (Figure 2, Table S3). More specifically, acetaldehyde and acrolein show a strong correlation $(r=0.804)$ with each other and with the other aldehydes. The weakest correlations are seen for formaldehyde with acetaldehyde $(p=0.614)$ and acrolein $(p=0.57)$, and they are even lower with the other aldehydes (Figure 2, Table S4).

TNCO Levels in Cigarette Mainstream Smoke. The same experimental setup was used to measure TNCO yields in the smoke of the different brands (Table 1, Table S1). The yields of TNCO measured with ISO differed with a maximum of $20 \%$ from the claimed TNCO levels printed on the cigarette package. In addition to the standard ISO regime, taping the cigarette filter resulted in an increase in TNCO yields (ISO-taped). The yields of tar increased between $18 \%$ and $939 \%$, nicotine yields between $0 \%$ and $148 \%$, and CO between $32 \%$ and $731 \%$ (ISO vs ISO-taped). When smoked with a higher puff volume and more frequent puffs using HCI-untaped, the TNCO yields at least doubled, compared to ISO. Using HCI resulted in higher tar and CO yields, respectively; varying between 5 and $413 \%$ and 9 and $264 \%$, compared to the HCI-untaped emissions. For nicotine, no clear increase was detected, with some brands even showing a decrease in nicotine values when HCI was used (HCI-untaped vs $\mathrm{HCI})$.
Influence of Smoking Protocol and Brand Characteristics, Especially Filter Ventilation. The filter tip ventilation differs between the brands in the range of 18.9-77.4\%. Taping the filter vents, thereby eliminating the tip filter ventilation to $0 \%$, as well as increasing puff volume and having a smaller puff interval both lead to an increase in aldehyde yields in cigarette smoke. More specifically, for most aldehydes, the impact of an intense smoking regime is at least two times larger than that of taping filter vents, except in the low-TNCO cigarettes, as shown in Figure 1. The low-TNCO brands Kent, Marlboro gold, Philip Morris One, and 1R5F have a filter tip ventilation in the range of $33.5-77.4 \%$. It is only for these highly ventilated low-TNCO cigarettes that closing ventilation holes for the ISO regime (ISO-taped) leads to a more increased aldehyde yield than using an intense smoking regime (HCI). For the most intense smoking condition (HCI with taped ventilation holes), the aldehyde yields even reached the levels of the other brands. This was observed in the cases of acrolein, acetaldehyde, acetone, crotonaldehyde, propionaldehyde, benzaldehyde, and 2,5-methylbenzaldehyde (Figure 1).

Toxicants per Nicotine Ratio per Brand. The difference in aldehyde yields per $\mathrm{mg}$ of nicotine per cigarette for the different cigarette brands under different smoking conditions is shown in Figure 3. The acrolein and acetaldehyde per nicotine ratio is larger in low-TNCO cigarettes. This trend observed for acetaldehyde, acrolein, formaldehyde, crotonaldehyde, butyraldehyde, and acetone (Figure 3 ) is also seen for the other aldehydes (not shown in Figure 3; propanal, butanal, isovaleraldehyde, $o-m$ - $p$-tolualdehyde, hexanal, and 2,5 dimethylbenzaldehyde). When smoking intensely $(\mathrm{HCI})$, regular cigarettes $(0.5-1.0 \mathrm{mg} / \mathrm{cig}$ according to the standard ISO regime) have comparable yields of aldehyde per mg of nicotine produced. In this case, taping has no increasing effect on the aldehyde production per mg of nicotine. The highest yields of aldehydes produced per mg of nicotine in smoke were measured in taped cigarettes when smoked with the ISO regime (ISO-taped). The largest difference is seen in low-TNCO cigarettes (ISO nicotine around $0.2 \mathrm{mg} / \mathrm{cig}$ ). In these low-TNCO cigarettes, up to six times more aldehyde production per mg nicotine is measured when 

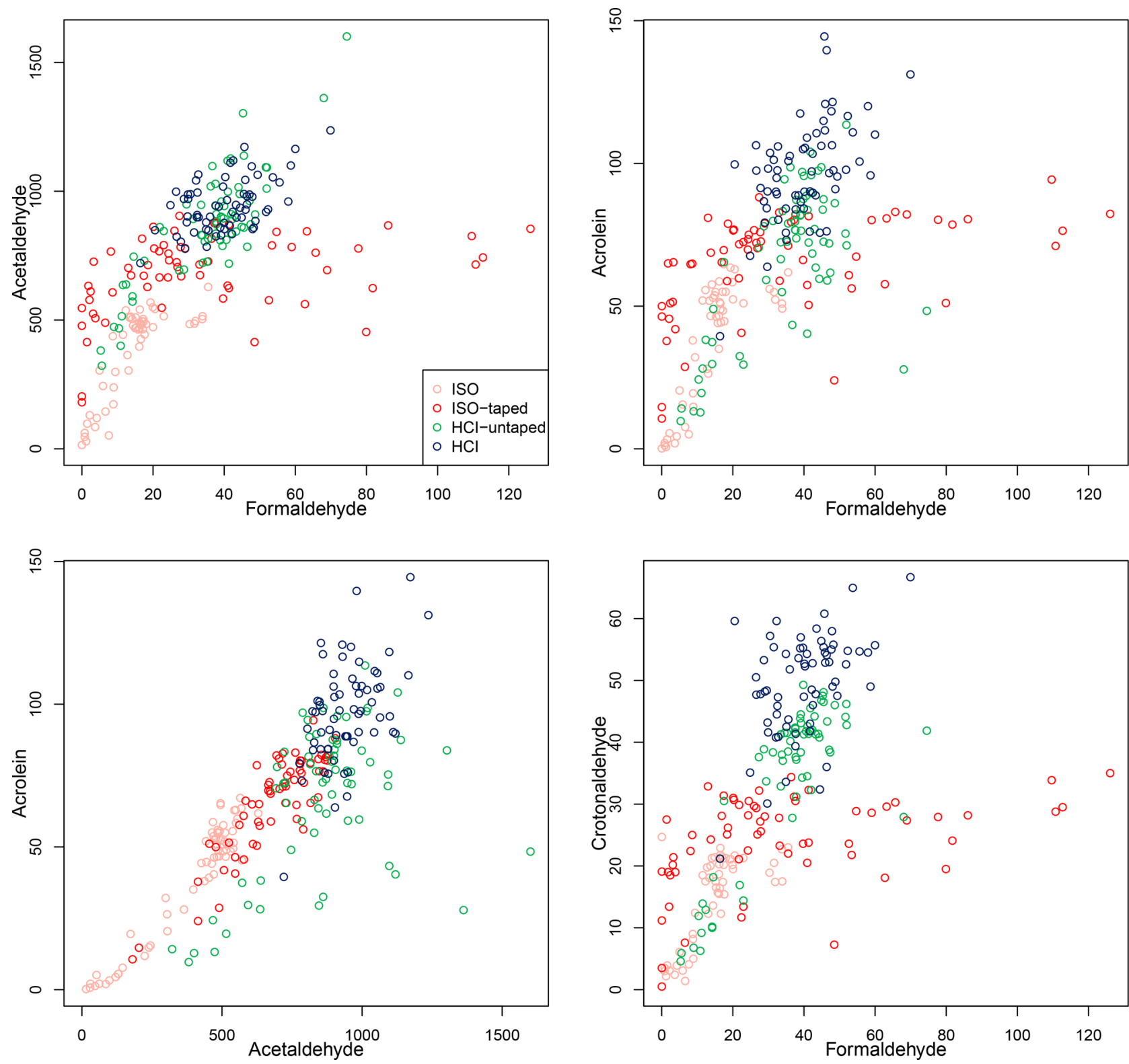

Figure 2. Correlation plots show the comparison of acetaldehyde vs formaldehyde (top left), acrolein vs formaldehyde (top right), acrolein vs acetaldehyde (bottom left), and crotonaldehyde vs formaldehyde (bottom right) (mean $\mu \mathrm{g} / \mathrm{cig}$ ). The replicates $(n=5)$ of the 13 cigarette brands are represented by dots in four different colors representing the four different smoking conditions. ISO regime is orange, ISO-taped is red, HCI-untaped is green, and $\mathrm{HCI}$ is blue.

comparing the least intense (ISO regime) with the most intense regime (HCI).

\section{DISCUSSION}

Aldehydes are an important class of toxicants in smoke, ${ }^{4,37}$ as has been recognized by WHO TobReg in their advice for mandating lower levels. ${ }^{2}$ While aldehyde levels in MSS have been studied before in several brands, ${ }^{11,16,38,39}$ this is the first study that systematically studies the influence of the smoking protocol on these levels. By using four different smoking protocols, ISO (standard without taping filter ventilation holes), ISO-taped, HCI-untaped, and HCI (standard with taping filter ventilation holes), and 11 brands, the effect of puff topography and filter ventilation can be compared. Furthermore, it was tested whether aldehyde levels correlate well with each other in order to test the hypothesis of Burns et al. that one or two components in a class can serve as indicator components. Finally, based on the findings, it was estimated whether brand differences impact a smoker's exposure to aldehydes.

Aldehyde Levels in Cigarette MSS. The method developed by the WHO TobLabNet, which is based on the work of Uchiyama et al., ${ }^{40}$ was used to measure aldehydes in 11 Dutch brands and two reference cigarettes in MSS generated using four different smoking protocols. Using the TobLabNet method, it was possible to measure aldehyde yields $(\mathrm{CV}<35.7 \%)$ comparable with previous studies of Counts et al., Uchiyama et al., Bodnar et al., Cheah et al., Reilly et al., and the CORESTA report $70+74 .^{11,16,38-40}$ Despite the difference in smoking regime and cigarette brands, nearly all of the aldehyde yields showed comparable patterns regarding their relative ratios. Individual aldehyde levels were highly correlated with each other, except for formaldehyde. ${ }^{39}$ More specifically, the cigarette 
Acetaldehyde to nicotine ratio



Formaldehyde to nicotine ratio

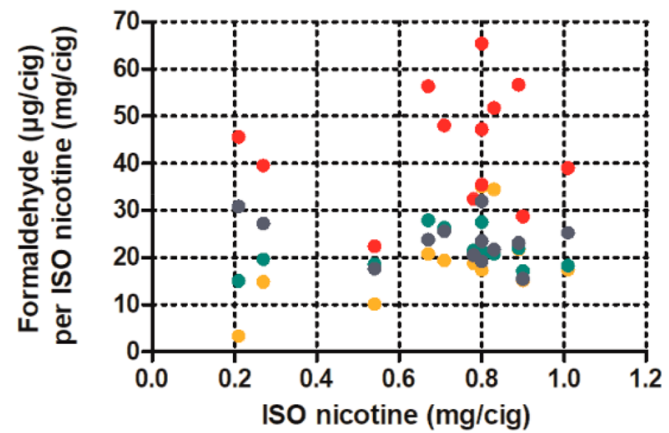

Butyraldehyde to nicotine ratio

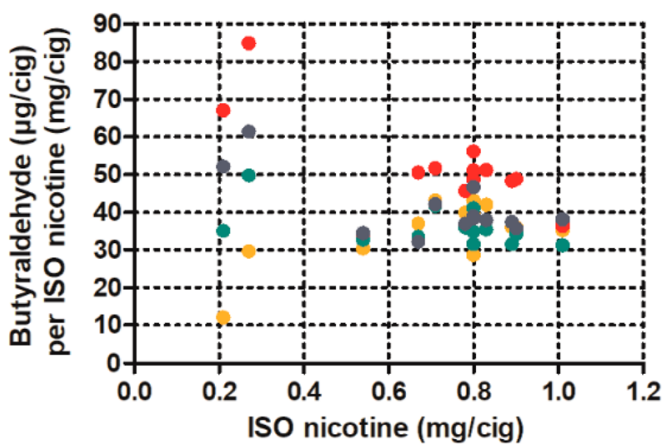

Acrolein to nicotine ratio

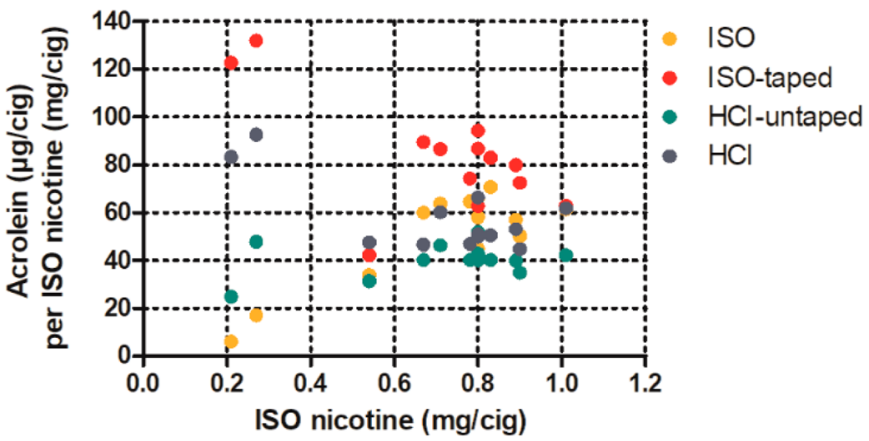

Crotonaldehyde to nicotine ratio

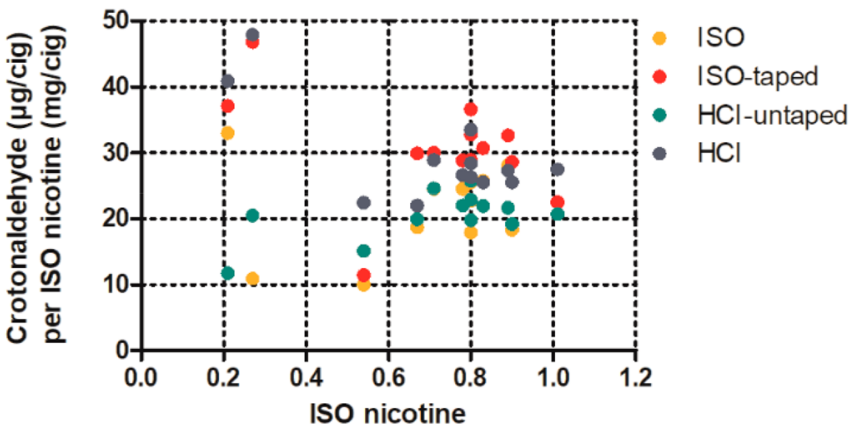

Acetone to nicotine ratio

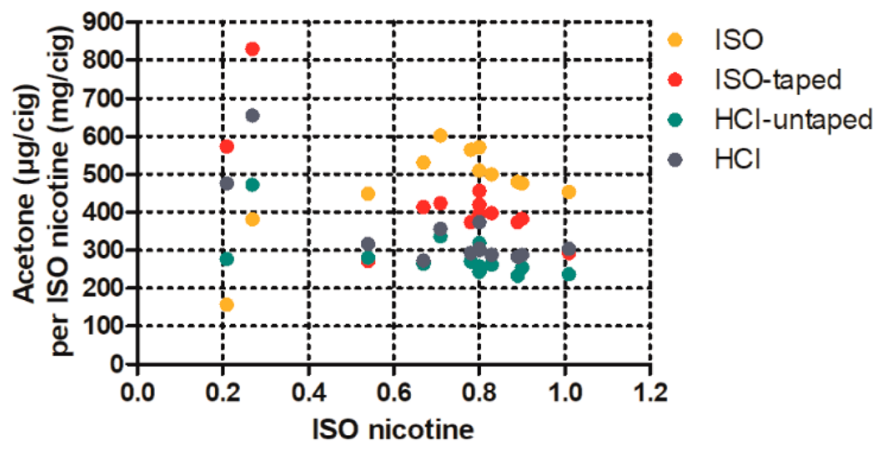

Figure 3. Toxicant-to-nicotine ratio. Acetaldehyde, acrolein, formaldehyde, crotonaldehyde, butyraldehyde, and acetone yields divided by nicotine $(\mathrm{mg} / \mathrm{cig})$ were measured using the four smoking conditions. On the $X$-axis, the nicotine $(\mathrm{mg} / \mathrm{cig})$ yields measured using the ISO method are shown to differentiate between the brands.

smoke yields of acrolein and acetaldehyde strongly correlated with a larger set of different aldehyde yields. ${ }^{41}$ This supports the proposal of Burns et al. to use a few aldehydes to represent the full class of aldehydes under different smoking conditions. ${ }^{2}$

A notable exception to this pattern is formaldehyde. Because formaldehyde is classified as a class 1 carcinogen by the IARC, ${ }^{42}$ there is no doubt about the wish to regulate this compound. However, the method used is not the most suitable one to measure formaldehyde. There are several differences between formaldehyde and the other aldehydes. Uchiyama et al. indicated that formaldehyde shows a wide analytical variation and problems with trapping. ${ }^{40}$ Our results also showed wide standard deviations, especially when the ISO regime was used. A more sophisticated method is therefore needed to measure formaldehyde. In particular, formaldehyde shows a different emission pattern under different smoking conditions and for different brands than the other 11 aldehydes. In addition, formaldehyde showed the poorest association with the other aldehydes ${ }^{39}$ and therefore seems less suitable as a representative of the aldehyde class.

Influence of Smoking Protocol. To test the effect of the smoking regime on the aldehyde yields, in combination with the cigarette design feature filter ventilation, both ISO and HCI regimes were used with and without taped filter ventilation holes.

The puff parameters of ISO and HCI differ in puff volume and interpuff interval, leading to additional smoke being produced for HCI. In the present study, these two conditions were tested with and without accounting for the influence of filter ventilation. Thus, ISO can be compared with HCI-untaped, and ISO-taped can be compared with HCI, allowing the effect of filter ventilation to be studied separately from the smoking topography. For both situations, the higher puff volume in combination with a shorter puff interval in HCI leads to a 
higher total puff volume and more puffs taken, when compared with ISO. Aldehyde yields in all cigarette brands increased when smoked according to HCI. Reilly et al. investigated the effect of puff parameters on the aldehyde yields in cigarette smoke and concluded that increased puff volume is the primary factor leading to higher aldehyde yields for the total volume per cigarette. ${ }^{39}$ As they found a similar influence of interpuff interval, puff flow, puff shape, and puff duration, they suggest that temperature changes play a minor role.

Influence of Filter Ventilation. In all cigarette brands, the highest yields of aldehydes in the cigarette smoke are measured when the filter ventilation holes of the cigarettes are taped during machine smoking. In addition to preventing dilution by sidestream air via filter vents, closing filter vents causes an increased burn rate of the cigarette, leading to more of the tobacco rod being consumed with every puff, thereby reducing the total burn time of the cigarette, as well as the number of puffs needed to completely smoke the cigarette. ${ }^{15}$ Aldehyde yields are increased during harsh burning conditions, which are gained by increasing puff volume or blocking ventilation. This explains why the effect of blocking ventilation holes is more pronounced for the ISO regime than the HCI regime. For the HCI regime, the harsh burning conditions due to intense smoking already led to an increase in aldehyde yields. The addition of blocking ventilation for the HCI regime leads to a less distinct increase in aldehyde yields, compared with the ISO regime. This also explains why the impact of ventilation blocking on aldehyde yields was the largest for those cigarettes with the highest initial ventilation (1R5F and Phillip Morris One), which was also found by Counts et al. ${ }^{16}$

Aldehyde-to-Nicotine Ratio per Brand. Brands show different aldehyde yields for the different smoking regimes when calculated as ratio to nicotine. In regular cigarettes, taping filter vents in ISO conditions (ISO-taped) led to the highest aldehyde-to-nicotine ratio. The increase in aldehyde-to-nicotine ratio is less distinct for the HCI regime when taping regular cigarettes. Thus, intensifying the smoking protocol by taking a puff more often and with a larger volume influences the aldehyde-to-nicotine ratio more than taping the filter vents. For the low-TNCO brands, the differences are even more prominent; these brands have a six times higher aldehyde production per $\mathrm{mg}$ nicotine for the intense smoking regimes, when compared to ISO. The fact that the aldehyde-to-nicotine ratio increased when low-TNCO cigarettes were smoked more intensely, similar to real smokers, implies that a smoker of low-TNCO cigarettes has an increased potential for higher aldehyde exposures as compared to a smoker of regular cigarettes.

Brand Differences and Filter Ventilation. Intensifying the smoking regime in combination with blocked filter ventilation substantially influences the aldehyde and TNCO yields in smoke. The filter tip ventilation is a design feature differing between brands. The filter tip ventilation for the 11 Dutch brands varied between $18 \%$ and $77 \%$. There is a trend that increasing filter ventilation leads to lower aldehyde yields, which is also found in literature. ${ }^{43}$ Filter tip ventilation higher than $60 \%$ significantly lowers the yields of nicotine, tar, and the aldehydes, as seen for the low-TNCO cigarettes. ${ }^{12}$

Aldehyde Exposure Estimation and Risk Assessment. On the basis of the aldehyde-to-nicotine ratios and a smoker's nicotine need, an estimation of aldehyde exposure when smoking according to different regimes can be made. Afternoon blood or plasma nicotine levels in smokers range from 10 to $50 \mathrm{ng} / \mathrm{mL}{ }^{44}$ To maintain this level, a smoker needs $1 \mathrm{mg}$ of nicotine every $2 \mathrm{~h}$ (taking a half-life of $1.5 \mathrm{~h}$ into account) and thus a total amount of $12 \mathrm{mg}$ per day. ${ }^{45}$ For example, based on the ISO regime, a Marlboro red $(0.8 \mathrm{mg} \mathrm{nic} / \mathrm{cig})$ smoker would need 15 cigarettes per day, compared to six cigarettes when smoking according to HCI ( $2 \mathrm{mg}$ nic/cig). This means a daily exposure to acetaldehyde of, respectively, $5.4 \mathrm{mg} /$ day (ISO $359.1 \mu \mathrm{g} / \mathrm{cig}$ ) or $5.6 \mathrm{mg} /$ day (HCI933.4 $\mu \mathrm{g} / \mathrm{cig}$ ). For the Philip Morris One, this means the smoker might need to smoke 60 cigarettes based on ISO $(0.2 \mathrm{mg} \mathrm{nic} / \mathrm{cig})$ and ten cigarettes for HCI $(1.2 \mathrm{mg} \mathrm{nic} / \mathrm{cig})$. The daily exposure to acetaldehyde is then $2.2 \mathrm{mg} /$ day (ISO $36.7 \mu \mathrm{g} / \mathrm{cig}$ ) and $10.6 \mathrm{mg} /$ day (HCI1055.6 $\mu \mathrm{g} / \mathrm{cig}$ ), a much larger difference between the two regimes than calculated for the Marlboro red.

Recent risk assessment studies show large adverse effects of aldehydes on human health. These methods combine machinegenerated yields with known chemical and toxicological properties. Risk assessment studies using inhalatory exposure risk factors were performed for the individual aldehydes. ${ }^{4,6,9}$ These computer models show a different order of chemical hazard ranking: acrolein > formaldehyde $>$ acetaldehyde ${ }^{9}$ compared to acrolein $>$ acetaldehyde $>$ formaldehyde. ${ }^{6}$ The ideal risk assessment methods in the case of cigarette smoke exposure use a mixture of aldehydes or assess a cumulative risk. The nonexistence of well-functioning mixture toxicity models is partly due to lack of inhalatory exposure assessments. The accurate smoke analysis data of the present study can advance inhalatory exposure assessments and the precision of mixture toxicity models. The changing levels in aldehydes due to choosing a different smoking regime may improve the accuracy of the models as designed, for example, by Corley et al., and other future comprehensive and specific risk assessments. ${ }^{9}$

Regulatory Implications. The ISO smoking regime is prescribed in the European Union Directive 2014/40/EU and used by manufacturers to declare TNCO yields. ${ }^{46}$ However, the standard ISO regime produced substantially lower aldehyde yields than the HCI regime, thus resulting in an underestimation of smokers exposure. Specifically, when regular and lowISO-TNCO-level cigarettes were smoked with the HCI regime or when filter ventilation holes were blocked (ISO-taped), overall aldehyde levels increased, as compared to the ISO regime. Since real smokers smoke more intensely than the ISO regime, the increased aldehyde levels when smoking more intensely or blocking filter vents can lead to higher aldehyde exposure. Thus, the present study results underline the need for a smoking regime that is more representative of actual human smoking behavior, like HCI, as also proposed in literature.

It has been proposed in the literature that toxicant levels should be regulated relative to $\mathrm{mg}$ of nicotine in $\mathrm{MSS}^{2}{ }^{2}$ The rationale for using exposures per $\mathrm{mg}$ of nicotine is that smokers need a certain amount of nicotine to sustain their addiction, and therefore adapt their smoking behavior in response to the cigarette design. The validity of this approach is demonstrated again by the present study results: for instance, the aldehyde levels increased at least 50\% more than the nicotine levels when a low-ISO-TNCO cigarette is smoked with the HCI regime, compared to a regular cigarette. Presuming that smokers smoke more intensely than the ISO regime, highly filter-ventilated cigarettes, often marketed as less harmful with a potentially reduced exposure claim, actually led to a higher exposure to toxic aldehydes. An alternative to regulation with respect to $\mathrm{mg}$ of nicotine is to regulate cigarette design characteristics. For instance, regulating cigarette design characteristics is a straightforward measure to prevent the high aldehyde-to-nicotine ratios 
that occur in cigarette smoke of low-ISO-TNCO cigarettes. A more specific regulatory approach involves setting maximum allowed aldehyde levels in cigarette smoke or a maximum exposure concentration range based on scientific arguments which are in line with previous proposals. ${ }^{2,47}$ As stated previously, the ISO regime underestimates the smokers' exposure to cigarette smoke toxicants, including aldehydes. The HCI regime is a better representative of human smoking and the associated aldehyde yields in the cigarette smoke of an actual smoker. To adapt smoking machine protocols so that they better represent typical exposure levels, human studies measuring natural smoking behavior are needed. Smokers have varying puff volumes and durations, and because each puff has its own characteristic chemical composition, this can lead to a complex smoke composition. ${ }^{17}$ The measured human smoking profile can be mimicked on the smoking machine to determine the exact associated aldehyde exposure. ${ }^{48}$ Specifically, a relevant set of aldehyde biomarkers can be measured in human fluids to link inhalatory exposure to internal dose. In the future, such data will show whether the reductions in machine-measured yields of specific constituents result in a reduction of exposure in smokers.

\section{ASSOCIATED CONTENT}

\section{S Supporting Information}

The Supporting Information is available free of charge on the ACS Publications website at DOI: 10.1021/acs.chemrestox.7b00342.

TNCO yields, aldehyde yields, statistical comparison values per individual aldehyde, and exact correlation factors of individual aldehydes (PDF)

\section{AUTHOR INFORMATION}

\section{Corresponding Author}

*E-mail: c.pauwels@maastrichtuniversity.nl.

ORCID ${ }^{\circ}$

Charlotte G.G.M. Pauwels: 0000-0001-6727-0804

\section{Funding}

This research did not receive any specific grant from funding agencies in the public, commercial, or nonprofit sectors.

\section{Notes}

The authors declare no competing financial interest.

\section{ACKNOWLEDGMENTS}

The author would like to thank Dr. Clifford H. Watson, Division of Laboratory Sciences, National Center for Environmental Health, U.S. Centers for Disease Control and Prevention (Atlanta, GA), and his personnel for undertaking the analysis of the filter ventilation for the 11 Dutch brands.

\section{ABBREVIATIONS}

TNCO, tar, nicotine, and carbon monoxide; WHO, World Health Organization; ISO, International Organization for Standardization; HCI, Health Canada Intense; IARC, International Agency for Research on Cancer; TobLabNet, Tobacco Laboratory Network

\section{REFERENCES}

(1) WHO. (2008) The Scientific Basis of Tobacco Product Regulation; second report of a WHO study group technical report series 951, World Health Organization, Geneva, Switzerland.
(2) Burns, D. M., Dybing, E., Gray, N., Hecht, S., Anderson, C., Sanner, T., O'Connor, R., Djordjevic, M., Dresler, C., Hainaut, P., Jarvis, M., Opperhuizen, A., and Straif, K. (2008) Mandated lowering of toxicants in cigarette smoke: a description of the World Health Organization TobReg proposal. Tob Control 17, 132-141.

(3) Hoffmann, D., Hoffmann, I., and El-Bayoumy, K. (2001) The less harmful cigarette: A controversial issue. A tribute to Ernst L. Wynder. Chem. Res. Toxicol. 14, 767-790.

(4) Talhout, R., Schulz, T., Florek, E., van Benthem, J., Wester, P., and Opperhuizen, A. (2011) Hazardous compounds in tobacco smoke. Int. J. Environ. Res. Public Health 8, 613-628.

(5) Rodgman, A., and Green, C. R. (2003) Toxic chemicals in cigarette mainstream smoke - hazard and hoopla. Beitraege zur Tabakforschung International 20, 481-545.

(6) Fowles, J., and Dybing, E. (2003) Application of toxicological risk assessment principles to the chemical constituents of cigarette smoke. Tob Control 12, 424-430.

(7) U.S. Food and Drug Administration. (2012) Harmful and potentially harmful constituents in tobacco products and tobacco smoke; established list, Vol. 77, No. 64, Department of Health and Human Services, Washington, U.S..

(8) U.S. Department of Health and Human Services. (2014) The Health Consequences of Smoking: 50 Years of Progress: A Report of the Surgeon General, Centers for Disease Control and Prevention, Atlanta, GA.

(9) Corley, R. A., Kabilan, S., Kuprat, A. P., Carson, J. P., Jacob, R. E., Minard, K. R., Teeguarden, J. G., Timchalk, C., Pipavath, S., Glenny, R. and Einstein, D. R. (2015) Comparative Risks of Aldehyde Constituents in Cigarette Smoke Using Transient Computational Fluid Dynamics/Physiologically Based Pharmacokinetic Models of the Rat and Human Respiratory Tracts. Toxicol. Sci. 146, 65-88.

(10) Cunningham, F. H., Fiebelkorn, S., Johnson, M., and Meredith, C. (2011) A novel application of the Margin of Exposure approach: segregation of tobacco smoke toxicants. Food Chem. Toxicol. 49, $2921-2933$

(11) Cheah, N. P. (2016) Volatile aldehydes in tobacco smoke: source fate and risk. Ph.D. Dissertation Thesis, Maastricht University, Maastricht, The Netherlands.

(12) Kozlowski, L. T., and O'Connor, R. J. (2002) Cigarette filter ventilation is a defective design because of misleading taste, bigger puffs, and blocked vents. Tob Control 11, i40-i50.

(13) Kozlowski, L. T., and O'Connor, R. J. (2000) Official cigarette tar tests are misleading: use a two-stage, compensating test. Lancet 355, $2159-2161$

(14) Marian, C., O’Connor, R. J., Djordjevic, M. V., Rees, V. W., Hatsukami, D. K., and Shields, P. G. (2009) Reconciling human smoking behavior and machine smoking patterns: implications for understanding smoking behavior and the impact on laboratory studies. Cancer Epidemiol., Biomarkers Prev. 18, 3305-3320.

(15) Hammond, D., Fong, G. T., Cummings, K. M., O'Connor, R. J., Giovino, G. A., and McNeill, A. (2006) Cigarette yields and human exposure: a comparison of alternative testing regimens. Cancer Epidemiol., Biomarkers Prev. 15, 1495-1501.

(16) Counts, M. E., Morton, M. J., Laffoon, S. W., Cox, R. H., and Lipowicz, P. J. (2005) Smoke composition and predicting relationships for international commercial cigarettes smoked with three machinesmoking conditions. Regul. Toxicol. Pharmacol. 41, 185-227.

(17) Adam, T., McAughey, J., Mocker, C., McGrath, C., and Zimmermann, R. (2010) Influence of filter ventilation on the chemical composition of cigarette mainstream smoke. Anal. Chim. Acta 657, $36-44$.

(18) Torikaiu, K., Uwano, Y., Nakamori, T., Tarora, W., and Takahashi, H. (2005) Study on tobacco components involved in the pyrolytic generation of selected smoke constituents. Food Chem. Toxicol. 43, 559-568.

(19) Chaiton, M. O., Collinshaw, N. E., and Callard, A. J. (2005) Smoker preference for "elastic cigarettes" in the Canadian cigarette market. Chronic Dis Can. 26, 20-24. 
(20) Hecht, S. S., Murphy, S. E., Carmella, S. G., Li, S., Jensen, J., Le, C., Joseph, A. M., and Hatsukami, D. K. (2005) Similar uptake of lung carcinogens by smokers of regular, light, and ultralight cigarettes. Cancer Epidemiol., Biomarkers Prev. 14, 693-698.

(21) Cummings, K. M., Hyland, A., Bansal, M. A., and Giovino, G. A. (2004) What do Marlboro Lights smokers know about low-tar cigarettes? Nicotine Tob. Res. 6, 323-332.

(22) Benowitz, N. L. (2001) Compensatory smoking of low-yield cigarettes. Smoking and Tobacco Control Monograph, No 13, U.S. Department of Health and Human Services, National Institutes of Health, National Cancer Institute, Bethesda, MD.

(23) Kozlowski, L. T., O’Connor, R. J., and Sweeney, C. T. (2001) Cigarette design. In Risks associated with smoking cigarettes having low machine-measured levels of tar and nicotine. Smoking and Tobacco Control Monograph, No 13, U.S. Department of Health and Human Services, National Institutes of Health, National Cancer Institute, Bethesda, MD.

(24) Roemer, E., Schorp, M. K., Piade, J. J., Seeman, J. I., Leyden, D. E., and Haussmann, H. J. (2012) Scientific assessment of the use of sugars as cigarette tobacco ingredients: a review of published and other publicly available studies. Crit. Rev. Toxicol. 42, 244-278.

(25) Adam, T., Mitschke, S., Streibel, T., Baker, R. R., and Zimmermann, R. (2006) Quantitative puff-by-puff-resolved characterization of selected toxic compounds in cigarette mainstream smoke. Chem. Res. Toxicol. 19, 511-520.

(26) Baker, R. R. (2006) The generation of formaldehyde in cigarettes-Overview and recent experiments. Food Chem. Toxicol. 44, $1799-1822$

(27) Seeman, J. I., Dixon, M., and Haussmann, H. J. (2002) Acetaldehyde in mainstream tobacco smoke: formation and occurrence in smoke and bioavailability in the smoker. Chem. Res. Toxicol. 15, 1331-1350.

(28) Talhout, R., Opperhuizen, A., and van Amsterdam, J. G. (2006) Sugars as tobacco ingredient: Effects on mainstream smoke composition. Food Chem. Toxicol. 44, 1789-1798.

(29) ISO 3402:2000. (2000) Tobacco and tobacco products atmosphere for conditioning and testing, International Organization for Standardization, Geneva, Switzerland.

(30) ISO 4387:2000/A1:2008. (2008) Cigarettes - Determination of total and nicotine-free dry particulate matter using a routine analytical smoking machine, International Organization for Standardization, Geneva, Switzerland.

(31) ISO 3308:2012. (2012) Routine analytical cigarette-smoking machine -definitions and standard conditions, International Organization for Standardization, Geneva, Switzerland.

(32) WHO Tobacco Laboratory Network. (2012) WHO SOP 01 Standard operating procedure for intense smoking of cigarettes, World Health Organization, Geneva, Switzerland.

(33) WHO Tobacco Laboratory Network. (2016) WHO SOP 08 Standard operating procedure for determination of aldehydes in mainstream cigarette smoke under ISO and intense smoking conditions, World Health Organization, Geneva, Switzerland.

(34) ISO 8454:2007/A1:2009. (2009) Cigarettes - Determination of carbon monoxide in the vapour phase of cigarette smoke - NDIR method, International Organization for Standardization, Geneva, Switzerland.

(35) ISO 10315:2014. (2014) Cigarettes - Determination of nicotine in smoke condensates - Gas-chromatographic method, International Organization for Standardization, Geneva, Switzerland.

(36) Norman, A. (1999) Cigarette design and materials. In Tobacco: production, chemistry, and technology (Davis, D. L., and Nielson, M. T., Ed.) pp 353-387, Blackwell Science, Oxford, U.K.

(37) Hoffmann, D., Djordjevic, M. V., and Hoffmann, I. (1997) The changing cigarette. Prev. Med. 26, 427-434.

(38) Bodnar, J. A., Morgan, W. T., Murphy, P. A., and Ogden, M. W. (2012) Mainstream smoke chemistry analysis of samples from the 2009 US cigarette market. Regul. Toxicol. Pharmacol. 64, 35-42.

(39) Reilly, S. M., Goel, R., Trushin, N., Elias, R. J., Foulds, J., Muscat, J., Liao, J., and Richie, J. P., Jr. (2017) Brand variation in oxidant production in mainstream cigarette smoke: Carbonyls and free radicals. Food Chem. Toxicol. 106, 147-154.

(40) Uchiyama, S., Tomizawa, T., Inaba, Y., and Kunugita, N. (2013) Simultaneous determination of volatile organic compounds and carbonyls in mainstream cigarette smoke using a sorbent cartridge followed by two-step elution. J. Chromatogr A 1314, 31-37.

(41) Reilly, S. M., Goel, R., Bitzer, Z., Elias, R. J., Foulds, J., Muscat, J., and Richie, J. P., Jr. (2017) Effects of Topography-Related Puff Parameters on Carbonyl Delivery in Mainstream Cigarette Smoke. Chem. Res. Toxicol. 30, 1463-1469.

(42) IARC. (2012) Monographs on the Evaluation of Carcinogenic Risks to Humans. Chemical Agents and Related Occupations Formaldehyde. 100F, International Agency for Research on Cancer, Lyon, France.

(43) Goel, R., Bitzer, Z., Reilly, S. M., Trushin, N., Foulds, J., Muscat, J., Liao, J., Elias, R. J., and Richie, J. P., Jr. (2017) Variation in Free Radical Yields from U.S. Marketed Cigarettes. Chem. Res. Toxicol. 30, 1038-1045.

(44) Benowitz, N. L. (2009) Pharmacology of nicotine: addiction, smoking-induced disease, and therapeutics. Annu. Rev. Pharmacol. Toxicol. 49, 57-71.

(45) Hukkanen, J., Jacob, P., 3rd, and Benowitz, N. L. (2005) Metabolism and disposition kinetics of nicotine. Pharmacol Rev. 57, $79-115$.

(46) European Union. (2014) Tobacco Products Directive (2014/40/ $E U)$, Official Journal of the European Union, Brussels, Belgium.

(47) Hecht, S. S. (2012) Research opportunities related to establishing standards for tobacco products under the Family Smoking Prevention and Tobacco Control Act. Nicotine Tob. Res. 14, 18-28.

(48) Shihadeh, A. L., and Eissenberg, T. E. (2011) Significance of smoking machine toxicant yields to blood-level exposure in water pipe tobacco smokers. Cancer Epidemiol., Biomarkers Prev. 20, 2457-2460. 